CARNETS DE Carnets de géographes

GÉOGRAPHES.

$1 \mid 2010$

Varia

\title{
Retour sur la création de Cybergéo
}

Entretien avec Denise Pumain

\section{Amandine Spire}

\section{(2) OpenEdition}

Journals

Édition électronique

URL : http://journals.openedition.org/cdg/1791

DOI : $10.4000 /$ cdg. 1791

ISSN : 2107-7266

Éditeur

UMR 245 - CESSMA

Référence électronique

Amandine Spire, «Retour sur la création de Cybergéo », Carnets de géographes [En ligne], 1 | 2010, mis en ligne le 01 octobre 2010, consulté le 14 septembre 2020. URL : http://journals.openedition.org/cdg/ 1791

Ce document a été généré automatiquement le 14 septembre 2020.

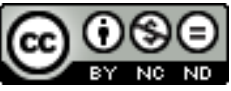

La revue Carnets de géographes est mise à disposition selon les termes de la Licence Creative Commons Attribution - Pas d'Utilisation Commerciale - Pas de Modification 4.0 International. 


\title{
Retour sur la création de Cybergéo
}

\author{
Entretien avec Denise Pumain
}

\author{
Amandine Spire
}

Entretien réalisé par Amandine Spire le 17 septembre 2010 à Paris.

A.s. Pourquoi avez-vous eu l'idée de créer une nouvelle revue?

D.P. La raison la plus importante, c'était une anticipation sur l'importance qu'allait prendre la documentation numérique dans l'activité scientifique. J'ai saisi au bond la proposition que me faisait un des ingénieurs du laboratoire de créer une revue à support numérique, d'accès libre, indépendante de toute maison d'édition, gratuite et qui permettait de mettre en ligne des articles comme une revue classique, mais aussi tout un ensemble de documents destinés à rendre service aux chercheurs. On a des listes de sites favoris, on publiait des listes de thèses, on a aussi lancé des débats...

L'autre raison qui motivait cette démarche, c'était une demande européenne. Depuis 1978, les géographes théoriques et quantitatifs (appellation d'origine assez large, allant de l'épistémologie au SIG en passant par la modélisation, et pas seulement) étaient réunis de manière informelle, mais à chaque fois avec 150, 200 personnes, tous les deux ans. Il s'agissait de géographes de tous les pays européens (avec quelques australiens et américains) réunis pour les recherches en cours, ce que vous appelez les recherches innovantes en géographie.

Ce groupe, bien que souhaitant rester informel dans son organisation, avait besoin d'un support de publication, de valorisation des présentations faites tous les deux ans. Et donc on chercha à créer un nouveau support : une revue. On a confié dans un premier temps la réalisation de cette ambition à un géographe anglais d'Oxford, Il est revenu avec un projet qui a vu le jour, un journal qui s'appelle maintenant Journal of Geographical Systems et qui au lieu d'être plurilingue comme on le souhaitait puisque c'était l'émanation d'un groupe européen, était entièrement monolingue (même pas de résumés dans une autre langue que l'anglais). Le premier numéro de ce journal était entièrement rédigé par des américains. On n'a pas énormément apprécié la mise en œuvre. Le pauvre collègue en question était fortement contraint par un éditeur privé. 
On m'a donc fortement sollicitée pour proposer une alternative à cette forme de publication internationale. Poussés par cette demande, nous avons créé cybergéo qui accueille des articles dans toutes les langues susceptibles de trouver des lecteurs (italien, espagnol, allemand, même hongrois). Les résumés sont toujours au moins dans deux langues dont l'anglais pour des questions de visibilité (l'anglais étant la langue la plus répandue...jusqu'à preuve du Chinois). Mise en ligne dès avril 1996, Cybergeo est ainsi la première revue scientifique entièrement électronique pour les sciences sociales et la géographie.

La forme de la revue était relativement peu classique puisqu'elle présentait des articles mais aussi d'autres types de textes. Au départ, avec accord de la bibliothèque de l'Institut de géographie, on mettait aussi en ligne les sommaires des autres revues, alors seulement sous format papier (dans la rubrique " revue des sommaires »).

A.s. Comment a été accueillie l'idée de cette revue, puis les premiers numéros?

D.P. Je ne sais pas si la revue électronique a inquiété les revues «papier » classiques. Il n'y a pas eu forcément de réaction, par exemple les Annales ne se sont pas exprimées. Moi-même, j'étais dans le comité de rédaction de L'Espace géographique, depuis 1977. À la demande de Roger Brunet, j'en assure la co-direction depuis 2003 avec Marie-Claire Robic. Il y a donc toujours eu le jeu de la co-habitation, mais au début j'étais agacée par certaines déclarations qui voulaient cantonner cybergéo à une "revue de brouillons» alors que d'emblée, pour durer et acquérir une visibilité internationale suffisante, on avait un comité de lecture extrêmement exigeant. Aujourd'hui, les articles publiés dans Cybergéo ont un niveau d'exigence comparable à celui de l'Espace géographique.

J'ai eu aussi la réaction de certains auteurs qui au départ estimaient moins valorisant de publier dans un support électronique que sur du papier. C'est en train de changer assez largement parce que le numérique est un support de valorisation qu'on ne peut refuser à la géographie. L'audience de Cybergéo est exponentielle. En termes de consultations, Cybergéo est en deuxième position dans le portail revues.org., après Nuovo mondo, revue publiée en espagnol. C'est important à savoir quand on s'interroge sur la place de la géographie dans les sciences humaines et sociales, les géographes étant souvent considérés comme des gens de sciences sociales de seconde zone. Or, au contraire, les géographes semblent très souvent à la pointe quand il s'agit d'assimiler des nouveautés conceptuelles ou techniques.

La nouveauté de Cybergéo résidait donc dans le numérique et dans la possibilité d'avoir un portail en plus de la revue offrant la possibilité de diversifier les rubriques. Nous avons toujours été ouverts à toutes les branches de la géographie (y compris à la géographie physique) sans aucun parti pris «idéologique». On veille à ce que les articles publiés soient en prise avec leur temps, et non la simple reproduction de quelque chose de déjà connu, et que les auteurs n'oublient pas de citer les références importantes du champ concerné.

A.s. Pour vous, que recouvre le terme de nouveauté en géographie?

D.P. Il y a tellement de formes de nouveauté en géographie...Tout d'abord, il y a la géographie de la "mise à jour ", c'est-à-dire le suivi de ce qui change dans le monde. On ne peut pas refuser complètement cette nouveauté-là. Quand on est géographe, on s'intéresse à ce qui apparaît, les transformations en cours dans une région du monde particulière, peu connue. Par exemple, le tourisme en Afrique du Sud. Des articles 
comme ça, on pourrait en écrire cinquante mille par jour. Cela s'apparente à du journalisme davantage qu'à une pratique scientifique de la géographie. Il s'agit donc d'une forme de nouveauté limitée, mais elle sert à tout le monde.

Il y a une autre manière d'apporter de la nouveauté : apporter un néologisme, un nouveau courant, des nouveaux mots, une nouvelle approche et ne la relier à rien de ce qui existe. Le faire en essayant à tout prix de se démarquer de ce qui a pu être fait avant. Ce n'est pas forcément satisfaisant...Parfois, un article peut défendre une idée intéressante, mais de manière affligeante, elle est présentée comme nouvelle de manière naïve sans que l'auteur ait conscience de tout ce qui a pu s'écrire et se travailler dans le champ investi. Je pense par exemple à ce qui se fait sur le capital géographique. Il pourrait y avoir un intérêt de nouveauté mais souvent ce n'est pas assez abouti.

Enfin, il y a une forme de nouveauté qui est beaucoup moins portée par le sens aigu de la distinction qui voyage dans nos sociétés médiatisées. Ce serait l'epsilon que l'on ajoute à un pan de la recherche, sur le plan documentaire, méthodologique, théorique. Autrement dit, il s'agit de la petite brique que l'on apporte au mur de la connaissance. Cela se produit lorsque la personne prend la peine de regarder ce qu'ont fait les autres et apporte une innovation sur le plan méthodologique, ou bien un test d'une idée déjà ancienne mais que l'on peut confronter à une grande base de données enfin constituées. C'est cela la nouveauté, dans le sens où cela participe à l'accumulation du savoir dont on a besoin dans nos disciplines pour exister, nous donner le droit d'enseigner un savoir à nos étudiants. Il faut s'enraciner dans ce savoir partagé et partageable. Le fond de la nouveauté, c'est ça, même si les deux autres acceptions [mentionnées plus haut] sont intéressantes. Chaque auteur dans sa vie écrit peut-être un article qui soit innovant dans ce sens-là car il y faut du temps, d'autant plus que l'on arrive jeune dans un monde trop vieux. Même s'il y a de nouvelles approches, le contenu sémantique des notions n'évolue jamais en rupture complète.

A.s. Pour vous, qu'est-ce qu'un objet de recherche original aujourd'hui en géographie?

D.P. Par exemple, on a publié un ensemble d'articles sur ce que Jérôme Monnet appelle l'ambulantage. C'est un type de commerce qui existe depuis le colportage médiéval. Mais évidemment dans les grandes villes contemporaines, cela prend des formes différentes du colportage médiéval ou de la vente à la camionnette qui existe encore dans nos campagnes reculées. On a accueilli ça comme une nouveauté qui implique d'innover en termes méthodologiques même si ce sont des outils déjà connus par les géographes travaillant sur les migrations (enquête à passage répété, mobilité de l'enquêteur et de l'enquêté, etc.). Il est nécessaire d'avoir recours à des analyses multi-échelles puisque les commerçants ambulants sont reliés à des réseaux internationaux.

Je pense aussi à une publication sur la vulnérabilité. C'est une approche du risque qui prend en compte la valeur des sociétés exposées. Par rapport à d'autres démarches plus classiques de l'analyse des risques, fondées uniquement sur la mesure d'un aléa physique, cela apporte un plus. Du point de vue des sciences sociales, on se trouve à l'interface de plusieurs disciplines. Il y a aussi des innovations pour lesquelles nous n'avons pas encore assez de publications, sur les modèles de simulation par exemple. Ce sont des outils qui nous permettent d'expérimenter des théories, de renouveler 
des expériences, en virtuel. Chaque fois que l'on a la possibilité de les nourrir un peu avec des données empiriques qui valident les hypothèses ou les règles que l'on a mises dans le modèle, on a des outils que l'on peut mettre entre les mains d'acteurs bien informés. Ce sont tous les domaines de la géographie qui apportent des innovations. Il y en a aussi dans une géographie qui serait très culturelle.

A.s. Qu'est ce qui serait donc « ancien » en géographie?

D.P. On ne va pas refaire de la géographie à tiroir. C'est une démarche que plus personne ne reconnaît ni n'admet car elle n'utilise pas les capacités de la géographie à lier et interroger les liaisons. Précisément, ce que la géographie a progressivement inventé ce sont des concepts pour relier ce qui était mis dans des tiroirs. Aussi, on ne rejette pas la forme du récit. Mais le récit donné pour lui-même, qui ne pose pas de question, sera nécessairement remis en question. Même en histoire, on ne produit pas un récit d'une séquence d'événements sans l'inscrire dans une réflexion ou une problématique. Enfin, une science se pose les questions qu'elle peut résoudre à un moment donné. C'est vrai aussi pour les sciences humaines et sociales. On le fait avec les moyens qu'on a de penser la géographie, la société, l'environnement. Un article qui se priverait de ces moyens de penser serait rejeté, discuté...

A.s. Comment accueillez-vous le projet éditorial des Carnets de géographes?

D.P. J'ai une réaction de sympathie et d'empathie. Je sais à quel point ce type de projet est mobilisateur et prend du temps. C'est un travail très important et parfois ingrat, notamment si vous prenez la peine de valoriser des productions inabouties. En même temps, je suis partagée car j'ai l'impression que s'épanouissent mille fleurs, comme aurait dit Mao, et que les potentielles forces éditoriales du milieu francophone, qui sont limitées, sont trop dispersées entre différents supports, ce qui les empêcherait ensemble d'acquérir une visibilité internationale suffisante. Il y a quantité de champs où la géographie française est dynamique, vivante, innovante. Il faut le faire savoir.

A.s. Pourquoi la géographie française serait-elle « résistante » à l'international ?

D.P. Il y a la question de la langue bien sûr. Écrire en langue étrangère revient à perdre un peu de son âme même si on y gagne en visibilité et parfois en simplicité d'expression (dans la mesure où les jeux sur les mots dans la langue maternelle peuvent être un obstacle à la communication scientifique). C'est une question délicate qui va compter de plus en plus, pas seulement pour l'évaluation, mais aussi pour le financement de la recherche, la manière de poser les questions... L'agenda scientifique est déjà dominé dans une certaine langue...

Je pense aussi qu'il faut essayer de s'enfermer dans la croyance à l'excellence nichée qui, autrefois, émergeait de toute façon. Je crois que cette excellence aura de plus en plus de peine à se faire voir. Autrement dit, avec le numérique, la mauvaise monnaie chasse la bonne. Je crois que nous sommes dans cette période-là. C'est pourquoi on essaie de créer des garde-fous. Cybergéo souhaite être une structure d'intermédiation entre les producteurs de sciences et les consommateurs/ou les lecteurs, qui garantit une certaine qualité, un niveau d'exigence. On a besoin de ces institutions intermédiaires entre l'individu et la société globale pour ne pas être trop victime du seul instrument qui est la visibilité médiatique, auto-proclamée, auto-entretenue. Il ne faut pas être naï. Une audience s'acquiert, se construit et se détruit 
indépendamment de la qualité du produit. La science est confrontée à ce défi. Malgré tout, les scientifiques ont assez de ressources pour résister.

A.s. Avez-vous l'impression que l'on pratique aujourd'hui une géographie différente de celle qui se pratiquait hier?

D.P. La réponse sera très différente suivant la pratique de géographie que l'on considère. D'un côté, les pratiques innovantes se sont diffusées auprès du grand public : géoportail, google map, GPS, etc. C'est un pan qui relevait de la cartographie, d'un savoir pointu, qui est devenu accessible au grand public, de plus en plus familier avec les cartes. On assiste à la valorisation d'outils ou de travaux autrefois confinés aux spécialistes de la géographie.

D'un autre côté, les thèses de doctorat servent à faire avancer la science. Je dirais que c'est même le seul moyen qu'ont les chercheurs de faire avancer la recherche sur un point qui nécessite une attention continue et ciblée étant donnée les contraintes institutionnelles actuelles. Les sujets que nous donnons aux étudiants sont nécessairement très spécialisés. Les recherches de doctorat sont inscrites dans un laboratoire qui donne à un étudiant les moyens bibliographiques, méthodologiques et les données qui vont lui permettre de traiter le sujet (pendant un ou deux ans) puis en général lors de la troisième ou quatrième année se produit l'innovation. L'étudiant rend compte de son sujet de manière nouvelle, apporte des résultats nouveaux, qui peuvent être des réponses à nos questions ou à d'autres questions. Le doctorant maîtrise alors mieux le sujet que ceux qui le lui ont donné.

A.s. Quel regard portez-vous sur la géographie explorée par les doctorants aujourd'hui ? Rejoint-elle certaines dimensions de la nouveauté et/ou de la marginalité évoquées précédemment?

D.P. Il y a le problème de faire la part de ce qui relève du doctorant et de l'encadrant quand le directeur s'approprie les sujets des doctorants. Dans le dialogue, il est difficile de savoir qui a amené quoi. C'est toujours une rencontre. Je peux vous donner un exemple à partir d'un sujet portant sur la possibilité d'explorer les données des téléphones mobiles pour étudier les mobilités en ville. Le sujet était conditionné par l'accès aux données. L'entrée a été suscitée par l'étudiant. On a va alors pouvoir construire la question à partir de ce qu'il est possible de faire avec la donnée. On ne sait pas ce qu'on en tirera. Il y a donc un risque. La nouveauté peut voir le jour grâce à un risque mais pas uniquement. Je pense à une thèse proposée par la RATP qui avait besoin d'un cartographe pour améliorer le système de représentation des statistiques concernant les incidents de petite délinquance (qui ne passent pas par la police) sur le réseau de métro et bus. Cela a fait l'objet d'une thèse en convention CIFRE mais on était dubitatif. Le sujet était a priori banal. L'étudiante a mené sa recherche et a construit un outil convenable pour la RATP. Dans le même temps, pour la thèse, elle a démontré des effets d'auto-corrélation spatiale. La doctorante a aussi mis en évidence que dans la manière dont les incidents étaient déclarés, le lieu n'était pas identifié assez précisément et donc que la base de données n'était pas utilisable au mieux. Le travail de recherche a été doublement satisfaisant puisque, outre la portée scientifique, la RATP, deux ans plus tard, a modifié la manière dont elle collecte/enregistre ses données géographiques. Les retombées de ce travail scientifique prouvent qu'il y a eu transmission d'un savoir qui a un intérêt, qui innove. Finalement, il est très difficile de prédéfinir l'aspect novateur d'un sujet. Parfois, il apparaît a posteriori. 
INDEX

Thèmes : Carnets de débats 